American Journal of Applied Sciences 7 (7): 943-948, 2010

ISSN 1546-9239

(C) 2010 Science Publications

\title{
HAZOP Analysis Management System with Dynamic Visual Model Aid
}

\author{
${ }^{1}$ Nan Bin Mad Sahar, ${ }^{2}$ Syahril Ardi, ${ }^{1}$ Suzuki Kazuhiko, ${ }^{1}$ Munesawa Yoshiomi and ${ }^{1}$ Minowa Hirotsugu \\ ${ }^{1}$ Division of Industrial Innovation Sciences, Department of Intelligent Mechanical Systems, \\ Graduate School Natural Science and Technology, Graduate School Natural Science and Technology, \\ Okayama University, Tsushima Naka 3-1-1, Okayama 700-8530, Japan \\ ${ }^{2}$ Polytechnic Manufacture Astra, \\ Faculty Production and Manufacture Process (Mechatronics) Engineering, Jakarta, Indonesia
}

\begin{abstract}
Problem statement: Ensuring safe operability and minimizing risk is the key component to prevent negative impact in all industries dealing with toxic, reactive, flammable and explosive. Hazard and Operability (HAZOP), a preliminary and systematic approach for identifying hazards has been unquestionably successful in reducing incidents by mitigating the consequence of major accident in the industries process facility. However, laborious work, time and cost are the shortcoming in performing and maintaining HAZOP analysis. Many research works over HAZOP automation available yet the traditional approach is still widely used by plant operators as it covers all part and aspect of a specific plant rather than generalizing to fit many plant types. Approach: In this study, we presented web based HAZOP analysis management system to help HAZOP team and related individual to perform revision, tracking and even complete HAZOP analysis without management bureaucracy. Besides depend solely to expert imaginative thinking of scenario using P\&ID, We added dynamic visual model to bring the system user a different view of consequent and subsequent to an accident and can be analyzed in three dimensional effects. This prevented miss looks due to 'paper based' view. Similarity matrix is responsible ensuring accuracy in analysis tracking. Reactor section in a hydrodesulphurization process was used as the case study to illustrate proposed system performance. Results: Developed system in average manages to reduce more than half the time required in performing HAZOP analysis compares to traditional method. Conclusion: This system expected to be the base engine for Virtual Reality simulator in analyzing accident caused by human factor.
\end{abstract}

Key words: Hazard identify, risk management, hydrodesulphurization, safety analysis

\section{INTRODUCTION}

Identifying hazards is fundamental for ensuring the safe design and system operation in process plants and other facilities. Several techniques are available to identify hazardous situations, all of which require their rigorous, thorough and systematic application by a multidisciplinary team of experts. Success rests upon first identifying and subsequently analyzing possible scenarios that can cause accidents with different degrees of severity. Without a structured identification system, hazards can be overlooked, so entailing incomplete risk-evaluations and potential loss. Identifying hazards even more critical for modern chemical manufacturing processes, which are often operated under extreme conditions to achieve maximum economic profit and sometimes have to undergo changes to meet customer demands. The importance of safety analysis in process operation is well recognized after an occurrence of several tragic accidents that could have been avoided if adequate process safety analysis had been done.

To ensure safe operation, Process Hazard Analysis (PHA) is very important to proactively identify the potential safety problems and recommend feasible mitigation actions. Among the available PHA techniques, Hazard and Operability (HAZOP) analysis is the most widely used in the Chemical Process Industry (CPI) (Cui et al., 2008). HAZOP analysis done by human teams, however, has the following shortcomings: Time consuming, laborious, expensive and inconsistent. To solve these problems, various models and/or rule-based HAZOP expert systems have

Corresponding Author: Nan Bin Mad Sahar, Division of Industrial Innovation Sciences, Department of Intelligent Mechanical Systems, Graduate School Natural Science and Technology, Okayama University, Tsushima Naka 3-1-1, Okayama 700-8530, Japan Tel: +81-86-251 Fax: +81-86-251-8059 
been developed during the last decade (Cheetam et al., 2006; Venkatasubramanian et al., 2000).

These systems, however, can only address processgeneric HAZOP analysis. Process-generic HAZOP analysis means that its reasoning logic can be applied to different processes while the non-process-generic HAZOP analysis means that its reasoning logic is process specific or plant specific. Generally analyses of deviations generated by using guidewords "other than", "as well as" and "part of" are non-process-generic. As a result, these kinds of deviations are hardly addressed in literature about HAZOP expert systems (Zhao et al., 2003; 2005). This is the reason why many plant operator running plant specific process having difficulty to integrated automated HAZOP in process hazard analysis.

To complete the HAZOP analysis of a typical chemical process, it takes about 1-8 weeks for a HAZOP team with 4-8 members. It is widely accepted that HAZOP analysis is an extremely time and effort consuming process. Moreover, the quality of HAZOP analysis depends on the knowledge and experience of the HAZOP team. Therefore, incompleteness and inconsistence usually are the drawbacks with regards to HAZOP done by human teams. Given the enormous amounts of time, effort and money involved in performing HAZOP, there exists considerable incentive to develop systems that can aid the process hazards analysis of chemical process plants. Here we list issue arise that can be improved to aid HAZOP analysis and management.

Management bureaucracy: In standard plant, process hazard analysis normally kept by the safety department or human resource department depending on production scale. Due to safety analysis documentation nature, the possibility, this documentation be revised or referred often are low. The troublesome procedure faced by the operator in terms of form filling, permission request for using or modifying leading obsolete analysis. The only possibilities this documentation be revised are during a safety audit which is depended on the plant management itself, which is every quarter year to one year.

Miss looks: Prior to HAZOP analysis, preparation including brainstorming, site visit and information gathering are conduct. During the analysis itself, HAZOP team will use $P$ and ID and their expert creative imagination to simulate the possible sequence and consequent. Often result to over looked mistakes where the location of real physical equipment is influencing the environment. For instant, heat from heat exchanger may leaked to a nearby container belong to totally different process line or overlapping pipes and equipments. This can only be notice by being on the site while doing the analysis.

Access limit: Analysis can only be done in certain part of factory such as meeting rooms which sometime inconvenient for the HAZOP team members. This might prevent competent inspiration.

In attempt to aid above problem, a semi automated safety management system called HAZOP analysis management system has been developed in this study.

\section{MATERIALS AND METHODS}

The proposed, HAZOP analysis management system is Web-based system utilizing Active Server Page (ASP) as the programming language. To meet the demand of space in storing the data and visual model, Microsoft SQL server 2005 is used. Models related to case study are developed and animated in Autodesk 3D MAX 9 exported to interactive format. We choose dwf format to future facilitate light file transfer web. Hydrodesulphurization process derived from training manual and safety procedure of standard VGO hydrodesulphurization. Key methods used are explained below.

HAZOP: Was introduced by Imperial Chemical Industries (ICI, UK) for identifying hazards in chemical plants in 1960s. HAZOP study is accomplished by a HAZOP team through a collective brainstorming effort that stimulates creativity and brings about new ideas of the potential hazards including their cause-effect relationships. Generally, the chemical process is divided into sessions called "analysis nodes" before the study. Then meaningful deviations in every analysis node are generated by combining process parameters and HAZOP guide words including MORE of, LESS of, NONE, REVERSE, PART of, AS WELL AS and OTHER THAN. For each deviation, the HAZOP team has to identify all of its credible causes and all of possible adverse consequences. Once the causes and consequences are recorded, the team has to list the existing safeguards for the identified hazards and give necessary recommendations accordingly for hazard mitigation if the required risk level cannot be achieved by the safeguards. The process is repeated deviation by deviation and node by node until the analysis of the whole process is completed. (López-Arévalo et al., 2007) The conventional HAZOP study procedure is presented in Fig. 1. 
Am. J. Applied Sci., 7 (7): 943-948, 2010

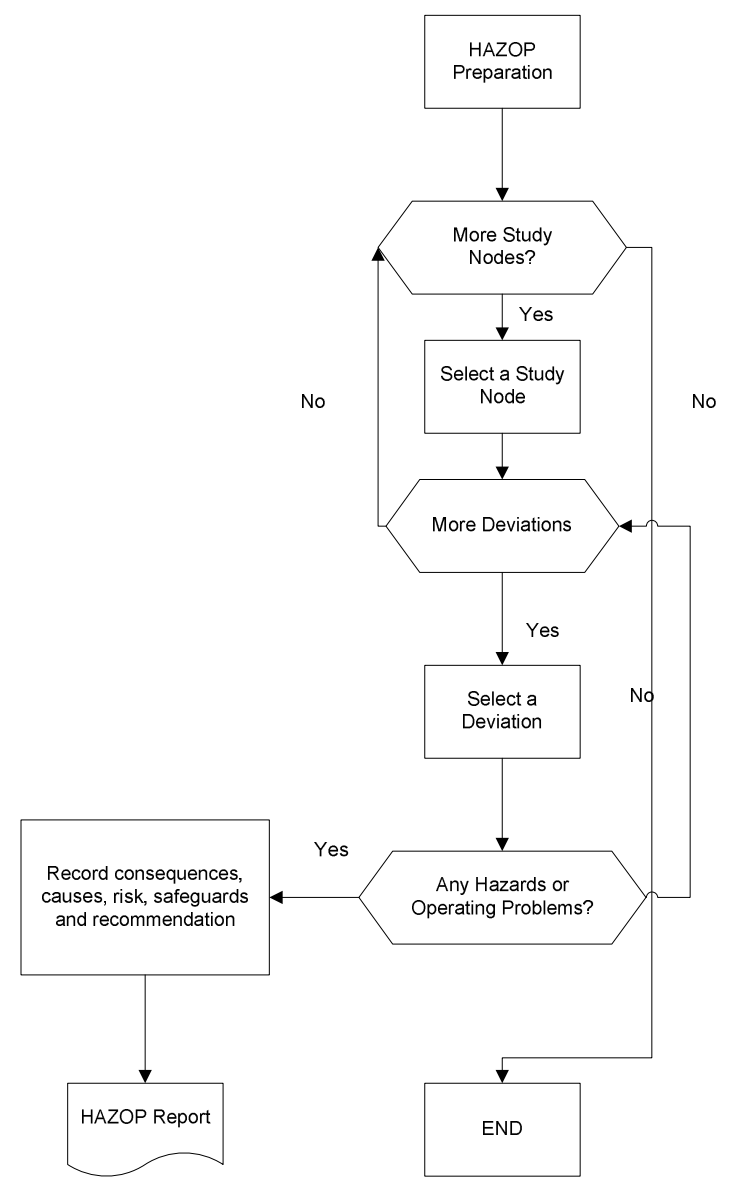

Fig. 1: HAZOP study procedure

Similarity check: In case matching process, the similarity rate between two set keyword is calculated. 1 is scored for complete similar cases, otherwise the gradient between 1 and 0 based on similarity rate (Pedersen et al., 2007). All numeric value is first transformed to non negative value. For term like, "Flow too fast" and "high flow" will be translated into "more flow" to ensure coherences. Temperatures different in range either plus one minus one is set as within the same case (Shimada et al., 1996). The similarity algorithm feature is extended-Euclidian algorithm. Suppose there are two attributes $A=\left[a_{1}, a_{2}\right], B=\left[b_{1}, b_{2}\right]$, then their similarity can be calculated as follows:

$\operatorname{Sim}(A, B)=\frac{\left(a_{1} b_{1}+a_{2} b_{2}\right)}{\max \left((a 1)^{2}+(a 2)^{2},(b 1)^{2}+(b 2)^{2}\right.}$

Work flow: By referring Fig. 2, the system starts with an authentication page. Users are filtered into three category base on the usability level:
- Admin-full access. Enable users to run complete HAZOP analysis. This includes modifying previous analysis, monitoring change and control over user access

- HAZOP team-limited access: Same as Admin except user management control capability

- Normal operator-view only. This access level gives permission only to view HAZOP analysis report

User then displayed with three analysis type they wish to do.

Predefined path analysis-offer user to select the path where the start node and the end node are already set according to running logic. Using the similarity matrix introduce above the most similar case are retrieved from the HAZOP case base database. In the same time visual model with the same attribute is also retrieved. This model is developed as similar as possible to the real equipment set as to assist HAZOP analyst. User than can revise and reused past HAZOP analysis if it is suitable. If an unsuitable, user can and creates a new analysis case.

Predefine process analysis-same as predefined path analysis. The different is users are offered starting node and end node based on a process rather than running logic.

New analysis-new analysis is where a complete HAZOP analysis of user selected start node and end node can be performed. Process type, process material and process equipment specification is provided to the user from the respective database. From here and forward, typical HAZOP study process starting by selecting guide word and deviation and continued to similarity matrix check as predefine process and predefine path analysis.

Case study: In order to illustrate the performance of our introduced system, we use Vacuum Gas Oil Hydrodesulphurization (VGO HDS) as the case study. Hydrodesulphurization is a catalytic chemical process widely used to remove Sulfur (S) from natural gas and from refined petroleum products such as gasoline or petrol, jet fuel, kerosene, diesel fuel and fuel oils (Cheetam et al., 2006). The purpose of removing the sulfur is to reduce the Sulfur dioxide $\left(\mathrm{SO}_{2}\right)$ emissions that result from using those fuels in automotive vehicles, aircraft, railroad locomotives, ships, gas or oil burning power plants, residential and industrial furnaces and other forms of fuel combustion. This case study is based on the standard VGO hydrodesulphurization plant model. 
Am. J. Applied Sci., 7 (7): 943-948, 2010

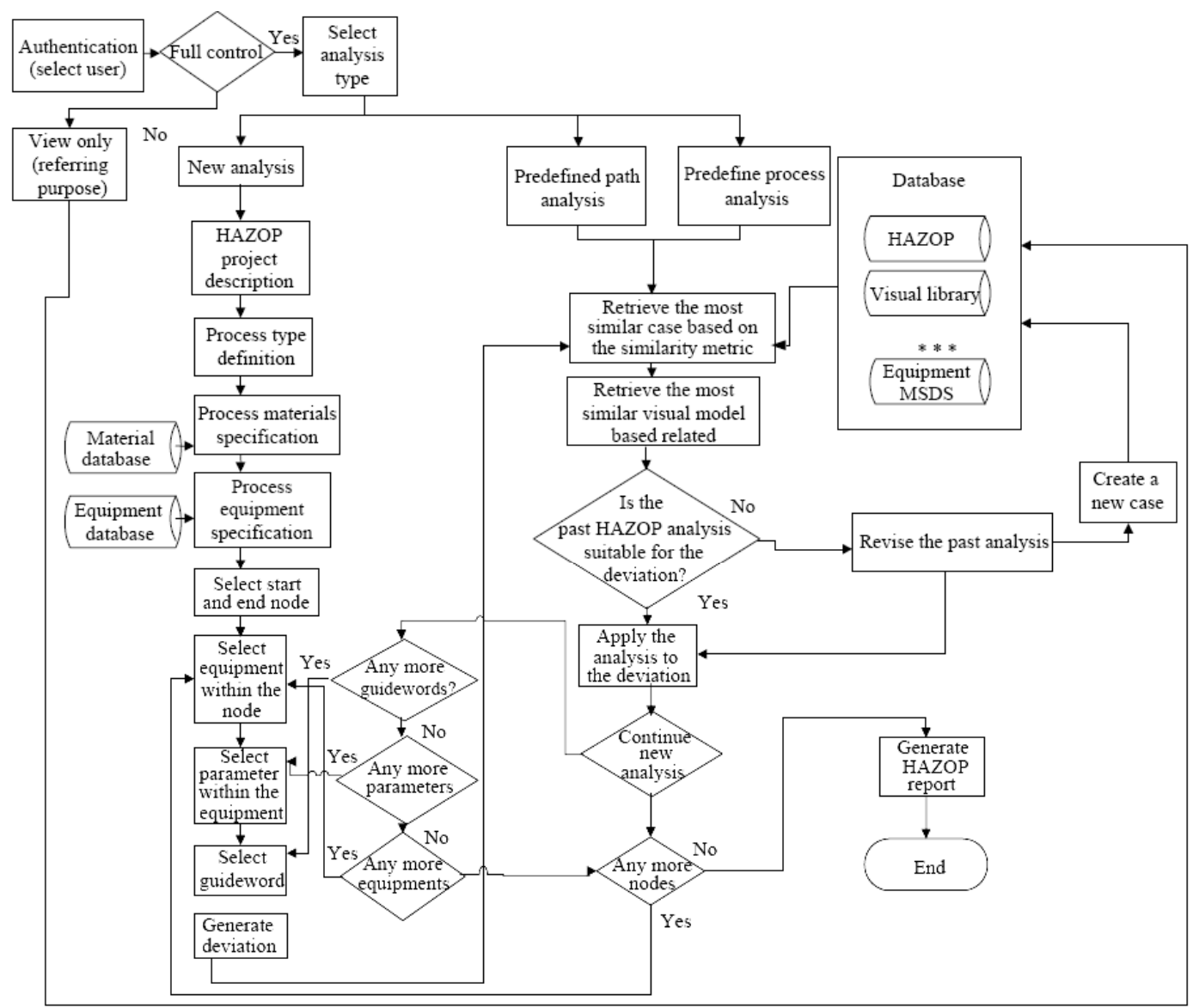

Fig. 2: Workflow diagram of HAZOP analysis management system

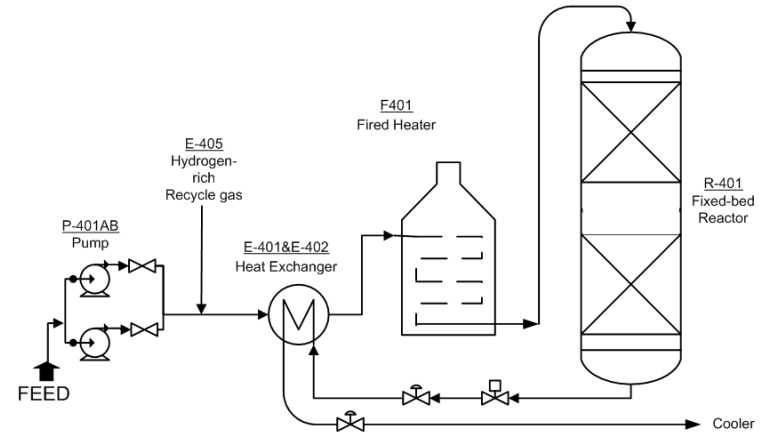

Fig. 3: Schematic diagram of a feed part of reactor section in VGO Hydrodesulphurization (HDS)

Referring to Fig. 3, the liquid feed Pumped (P$401 \mathrm{AB})$ up to the required elevated pressure and is joined by a stream of hydrogen-rich recycle gas (E405). The resulting liquid-gas mixture is preheated by flowing through a heat Exchanger (E-401). The preheated feed then flows through a Fired heater (F401) where the feed mixture is totally vaporized and heated to the required elevated temperature before entering the Reactor (R-401) and flowing through a fixed-bed of catalyst where the hydrodesulphurization reaction takes place.

The hot reaction products are partially cooled by flowing through the heat Exchanger (E-401) where the reactor feed was preheated and then flows through a water-cooled heat exchanger.

\section{RESULTS}

Figure 4 show the main page during revising analysis result. User name and access level is display on top along with date and time. Any changes by the user will be timestamp using above time. This enables future monitoring and tracking process of fault analysis. Two icons beside the analysis type and analysis path represent Piping and Instrumentation Diagram (P and ID) and Visual model as in Fig. 5. The page is divided into two parts, HAZOP analysis results and the HAZOP system analysis result. 


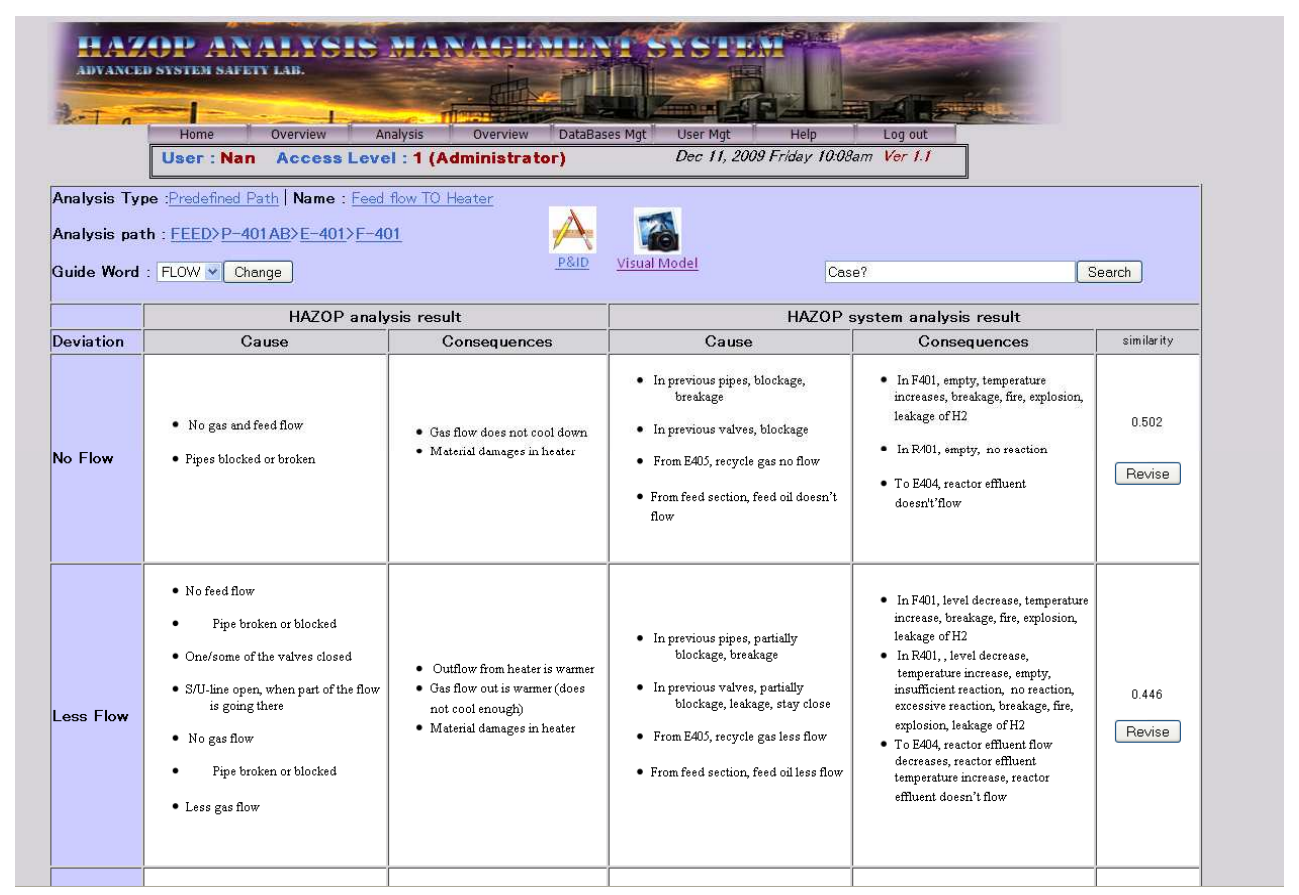

Fig. 4: Snapshot of a main page during revising analysis result

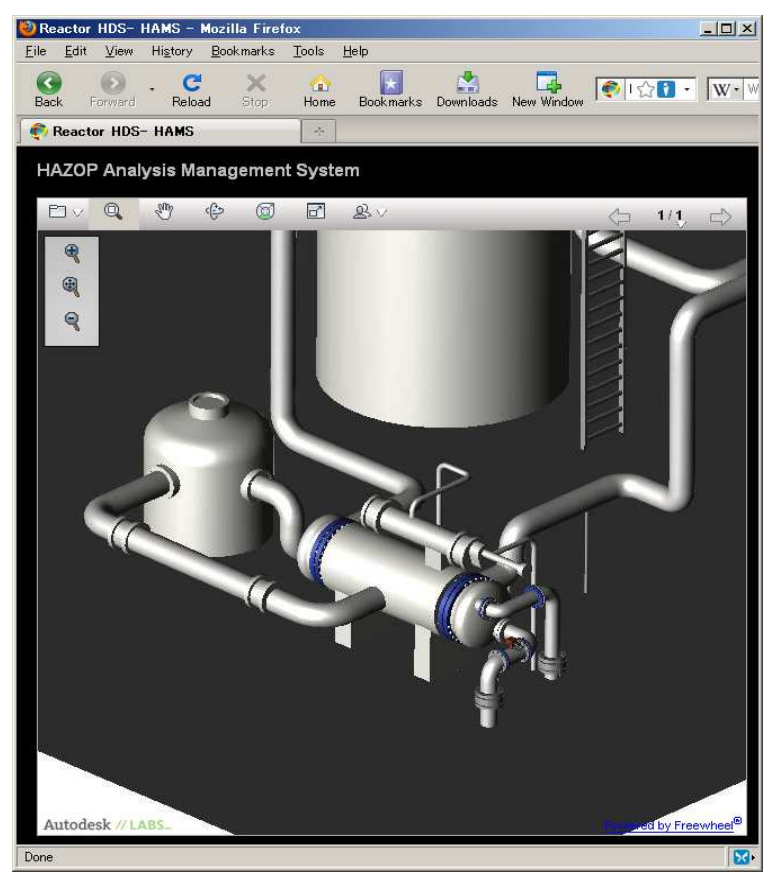

Fig. 5: Visual model of HDS reactor part

Keywords attach to stored cases compared with user analysis, similarity score display right. The pages will bring the user to editing page, should additional information or new analysis exist.
Table 1: Comparison time consumed for HAZOP analysis based on guide word between manual and proposed system supported analysis

\begin{tabular}{lccl}
\hline Guide word & Manual* $(\mathrm{min})$ & System $(\mathrm{min})$ & Time saved \\
\hline Flow & 128 & 45 & 2.8 \\
Temperature & 90 & 39 & 2.3 \\
Hydrogen consistency & 240 & 155 & 1.5 \\
Pressure & 65 & 42 & 1.5
\end{tabular}

*: Time recoded to the nearest minute and 2 min error margin

HAZOP analysis is carried out in both conventional ways and using proposed system. Time consumed for each are calculated.

Table 1 show the comparison time consumed. Time manage to be save depend on process complexity involving the guide word. On average, proposed system improves $35-54 \%$ analysis time.

\section{DISCUSSION}

The advantages the proposed system brings in aiding HAZOP analysis:

- Eliminate management bureaucracy problem. Where record tracking can be done without having to fill forms or meeting with HR department to get permission. All modification with timestamp are recorded for monitoring purpose. Access and modification permission are set and limit by user type 
- Giving analysis different perspective of creative imagination. By using visual model, the system allow analysts to navigate different angle and proportions eliminate blind spot

However, the lack of ontology to support similarity check module remain in this study. Without complete ontology, it is difficult for the system algorithm to translate cases with similar meaning. Standardization during registering new cases might help reduce problems but prevent description accuracy of cases. The extended-Euclidian algorithm used in the system only find similarities by comparing keywords consists between the cases. Ability to evaluate sentences (case) with high meaning similarity is considered for future research.

\section{CONCLUSION}

We present web based HAZOP analysis management system to help HAZOP team and related individual to perform revision, tracking and complete HAZOP analysis without management bureaucracy which increase time consumed during the process. Besides depend solely to expert imaginative thinking of scenario using $\mathrm{P}$ and ID, the dynamic visual model aid $\mathrm{g}$ the system user a different proportions of consequent and subsequent to an accident and able to analyses in three dimensional effects. This prevents miss looks due to blind spot happened during site visit and HAZOP preparation. Similarity matrix used, responsible ensuring accuracy in analysis tracking minimize case retrieving leading to time saving. We design this system to be the base for future research, which expected to be integrated with full functional visual reality simulations that add human factor analyzing.

\section{ACKNOWLEDGMENT}

The researchers are grateful for the financial and equipment support provided by Okayama University.

\section{REFERENCES}

Cheetam, W., S. Shiu, O. Rosina and O. Weber, 2006. Soft case-based reasoning. Knowl. Eng. Rev., 20: 267-269.

Cui, L., J. Zhao, T. Qiu and B. Chen, 2008. Layered digraph model for HAZOP analysis of chemical processes. Process Safe. Prog., 27: 293-305. DOI: 10.1002/prs.10266

López-Arévalo, I., R. Ban Ares-Alcantara, A. Aldea, A. Rodriguez-Martinez and L. Jimenez, 2007. Generation of process alternatives using abstract models and casebased reasoning. Comput. Chem. Eng., $\quad 31$ : 902-918. DOI: 10.1016/j.compchemeng.2006.08.011

Pedersen, T., S.V.S. Pakhomov, S. Patwardhan and C. Chute, 2007. Measure of semantic similarity and relatedness in biomedical domain. J. Biomed. Inform., $\quad 40$ : $288-299 . \quad$ DOI: 10.1016/j.jbi.2006.06.004

Shimada, Y., K. Suzuki and H. Sayama, 1996. Computer-aided operability study. Comput. Chem. Eng., 20: 905-913. DOI: 10.1016/00981354(95)00187-5

Venkatasubramanian, V., J. Zhao and S. Viswanathan, 2000. Intelligent systems for HAZOP analysis of complex process plants. Comput. Chem. Eng., 24: 2291-2302. DOI: $10.1016 /$ S00981354(00)00573-1

Zhao, C., M. Bhushan and V. Venkatasubramanian, 2003. Roles of ontology in automated process safety analysis. Comput. Aid. Chem. Eng., 14: 341-346. DOI: 10.1016/S1570-7946(03)80138-4

Zhao, C., M. Bhushan and V. Venkatasubramanian, 2005. PHASUITE: An automated HAZOP analysis tool for chemical processes part I. Knowl. Eng. Framework Process Safe. Environ. Prot., 83: 509-532. DOI: $10.1205 /$ psep.04055 